\title{
TAUBERIAN THEOREMS FOR ABSOLUTE SUMMABILITY
}

J. S. RATTI

1.1. Let $\sum a_{n}$ be an infinite series, and let $\left\{\lambda_{n}\right\}$ be an arbitrary sequence of positive numbers tending to infinity with $n$, such that

$$
1 \leqq \lambda_{1}<\lambda_{2}<\cdots
$$

We write

$$
\begin{aligned}
& A_{\lambda}^{k}(x)=\sum_{\lambda_{n}<x}\left(x-\lambda_{n}\right)^{k} a_{n}=\int_{1}^{x}(x-t)^{k} d A_{\lambda}(t), \\
& A_{\lambda}^{0}(x)=A_{\lambda}(x)=\sum_{\lambda_{n}<x} a_{n}, \\
& A_{\lambda}^{k}(x)=0 \quad \text { for } x \leqq 1 \text { and } k>-1 .
\end{aligned}
$$

We also write

$$
B_{\lambda}^{k}(x)=\sum_{\lambda_{n}<x}\left(x-\lambda_{n}\right)^{k} \lambda_{n} a_{n} .
$$

The series $\sum a_{n}$ is said to be summable $|R, \lambda, k|_{m}, k>0, m \geqq 1$, $k>1-1 / m$, if

$$
\int_{1}^{\infty} x^{m-1}\left|\frac{d}{d x} x^{-k} A_{i}^{k}(x)\right|^{m} d x<\infty .
$$

The case $\lambda_{n}=n$ of this definition is given in [1] where it is shown why the additional restriction $k>1-1 / m$ is necessary.

The series $\sum a_{n}$ is said to be summable $|A, \lambda|_{m}, m \geqq 1$, if the series $f(x)=\sum a_{n} \exp \left[-\lambda_{n} x\right]$ converges for $x>0$ and

$$
\int_{0}^{\infty}\left(1-e^{-x}\right)^{m-1}\left|f^{\prime}(x)\right|^{m} d x<\infty,
$$

[8, Theorem 2].

It is easily seen that for $m=1$, summability $|A, \lambda|_{m}$ and summability $|R, \lambda, k|_{m}$ are the same as summability $|A, \lambda|$ [9] and summability $|R, \lambda, k|[2]$ respectively. Borwein [1] has shown that for $\lambda_{n}=n$ summability $|R, \lambda, k|_{m}$ of $\sum a_{n}$ is equivalent to its absolute Cesàro summability with index $m$.

1.2. Hyslop [6] has established the following Tauberian theorem for absolute summability.

Received by the editors August 22, 1966. 
TheоReм A. If $\sum a_{n}$ is summable $|A|$ and $\sum \Delta\left(n a_{n}\right)$ is summable $|C, k+1|$, where $k \geqq 0$, then $\sum a_{n}$ is summable $|C, k|$.

Flett [3] generalized Theorem A for index $m$ and proved the following theorem.

THEOREM B. Let $m \geqq 1, k>-1$. If $\sum a_{n}$ is summable $|A|_{m}$ and if also $\sum \Delta\left(n a_{n}\right)$ is summable $|C, k+1|_{m}$, then $\sum a_{n}$ is summable $|C, k|_{m}$.

The object of this paper is to obtain an analogue of Theorem B involving the extended definitions of absolute Abel and absolute Riesz summability referred to above as summability $|A, \lambda|_{m}$ and summability $|R, \lambda, k|_{m}$.

I wish to express my appreciation to Professor D. Waterman for his guidance during the preparation of this paper. I also thank the referee for his valuable suggestions, especially Theorem 3.

2.1. We prove the following theorems.

THEOREM 1. If (i) $\sum a_{n}$ is summable $|A, \lambda|_{m}, m \geqq 1$, then a necessary and sufficient condition for the given series $\sum a_{n}$ to be summable $|R, \lambda, k|_{m}$ is that

(ii) $\int_{0}^{\infty} x^{m-1}\left|(d / d x) x^{-k-1} B_{\lambda}^{k}(x)\right|^{m} d x<\infty$, where $k>1-1 / m$.

TheOREM 2. If (i) $x^{-1} B_{\lambda}^{0}(x)$ is of bounded variation in $(0, \infty)$ and (ii) the series $\sum a_{n} \exp \left[-\lambda_{n} x\right]$ is convergent for all $x>0$ and its sum $f(x)$ is of bounded variation in $(0, \infty)$, then $\sum a_{n}$ is absolutely convergent. Moreover, the conditions (i) and (ii) are also necessary for the absolute convergence of $\sum a_{n}$.

2.2. We require the following lemmas for the proof of our theorem.

LEMMA 1 [5]. If $k>0$, then

$$
d / d x\left(x^{-k} A_{\lambda}^{k}(x)\right)=k x^{-k-1} B_{\lambda}^{k-1}(x) .
$$

LEMмA 2 [2]. If $f(x)=\sum a_{n} \exp \left[-\lambda_{n} x\right]$ converges for $x>0$, then for $k \geqq 0$ we have

$$
f(x)=\frac{x^{k+1}}{\Gamma(k+1)} \int_{0}^{\infty} A^{k}(t) e^{-x t} d t .
$$

Lemma 3. For (i) $\sum a_{n}$ to be summable $|R, \lambda, k|_{m}$ it is necessary and sufficient that

(ii) $\sum a_{n}$ be summable $|R, \lambda, k+1|_{m}$ and

(iii) $\int_{0}^{\infty} x^{m-1}\left|(d / d x) x^{-k-1} B_{\lambda}^{k}(x)\right|^{m} d x<\infty$, where $m \geqq 1, k>1-1 / m$.

Proof of Lemma 3. (a) Let $m>1$. Since $A_{\lambda}^{k}(x)=0$ for $x \leqq 1$, we write 


$$
\int_{0}^{\infty} x^{m-1}\left|\frac{d}{d x} x^{-k} A_{\lambda}^{k}(x)\right|^{m} d x=\int_{1}^{\infty} x^{m-1}\left|\frac{d}{d x} x^{-k} A_{\lambda}^{k}(x)\right|^{m} d x .
$$

We have

$$
\frac{d}{d x}\left(x^{-k-1} A_{\lambda}^{k+1}(x)\right)=(k+1) x^{-k-1} A_{\lambda}^{k}(x)-(k+1) x^{-k-2} A_{\lambda}^{k+1}(x) .
$$

Using Lemma 1, we get

$$
x^{-k} A_{\lambda}^{k}(x)=x^{-k-1} A_{\lambda}^{k+1}(x)+x^{-k-1} B_{\lambda}^{k}(x) .
$$

Therefore

$$
\begin{aligned}
\int_{\theta}^{\infty} x^{m-1}\left|\frac{d}{d x} x^{-k} A_{\lambda}^{k}(x)\right|^{m} d x \leqq & M \int_{0}^{\infty} x^{m-1}\left|\frac{d}{d x} x^{-k-1} A_{\lambda}^{k+1}(x)\right|^{m} d x \\
& +M \int_{0}^{\infty} x^{m-1}\left|\frac{d}{d x} x^{-k-1} B_{\lambda}^{k}(x)\right|^{m} d x .
\end{aligned}
$$

The sufficiency follows, since by (ii) and (iii) the right-hand side is finite. Throughout this paper $M$ denotes a positive constant which is not necessarily the same at every occurrence.

Since summability $|R, \lambda, k|_{m}$ implies summability $|R, \lambda, k+1|_{m}$ [7], using (2.2.1) we get

$$
\begin{aligned}
\int_{0}^{\infty} x^{m-1}\left|\frac{d}{d x} x^{-k-1} B_{\lambda}^{k}(x)\right|^{m} d x \leqq & M \int_{0}^{\infty} x^{m-1}\left|\frac{d}{d x} x^{-k} A_{\lambda}^{k}(x)\right|^{m} d x \\
& +M \int_{0}^{\infty} x^{m-1}\left|\frac{d}{d x} x^{-k-1} A_{\lambda}^{k+1}(x)\right|^{m} d x .
\end{aligned}
$$

The right-hand side is again finite, the lemma follows.

(b) For $m=1$, the lemma follows at once from (2.2.1) and the first theorem of consistency [2] for $|R, \lambda, k|$ summability of $\sum a_{n}$.

REMARK. It should be pointed out that Lemma 3 continues to hold when $m=1$ and $k=0$. It is easy to see that (2.2.1) holds for $k=0$. Since

$$
\sum_{\lambda_{n}<x} a_{n}-\sum_{\lambda_{n}<x}\left(1-\frac{\lambda_{n}}{x}\right) a_{n}=x^{-1} B_{\lambda}^{0}(x) .
$$

2.3. Proof of Theorem 1. The necessity part of the theorem follows from Lemma 3. Therefore we have only to prove that conditions (i) and (ii) are sufficient for the summability $|R, \lambda, k|_{m}$ of the given series. In view of Lemma 3 , it is sufficient that the given series be summable $|R, \lambda, k+1|_{m}$, i.e. 


$$
I=\int_{0}^{\infty} x^{m-1}\left|(k+1) x^{-k-2} B_{\lambda}^{k}(x)\right|^{m} d x<\infty .
$$

By Lemma 2, on account of hypothesis (i), we have

$$
f^{\prime}(x)=-\frac{x^{k+1}}{(k+1)} \int_{0}^{\infty} B_{\lambda}^{k}(t) e^{-x t} d t .
$$

Again by hypothesis (i)

$$
\int_{0}^{\infty}\left(1-e^{-x}\right)^{m-1}\left|f^{\prime}(x)\right|^{m} d x<\infty,
$$

and by second mean value theorem, for some $R, 0<R<\infty$, this in tegral is equal to

$$
\begin{aligned}
& \int_{0}^{R}\left(e^{x}-1\right)^{m-1}\left|f^{\prime}(x)\right|^{m} d x \\
& \quad=\int_{1 / R}^{\infty}\left(e^{1 / x}-1\right)^{m-1}\left|f^{\prime}\left(\frac{1}{x}\right)\right|^{m} x^{-2} d x>\int_{1 / R}^{\infty} x^{-m-1}\left|f^{\prime}\left(\frac{1}{x}\right)\right|^{m} d x .
\end{aligned}
$$

Hence it will suffice to show that

$$
J=\int_{1 / R}^{\infty}\left|(k+1) x^{-k-1-1 / m} B_{\lambda}^{k}(x)-\frac{x^{-k-2-1 / m}}{\Gamma(k+1)} \int_{0}^{\infty} B_{\lambda}^{k}(t) e^{-t / x} d t\right|^{m} d x<\infty .
$$

Writing $g(x)$ for $x^{-k-1} B_{\lambda}^{k}(x)$, we have

$$
\begin{aligned}
J \leqq M \int_{0}^{\infty} x^{m-1} \mid g(x) x^{-k-3} \frac{1}{\Gamma(k+2)} & \int_{0}^{\infty} t^{k+1} e^{-t / x} d t \\
& -\left.\frac{x^{-k-3}}{\Gamma(k+2)} \int_{0}^{\infty} B_{\lambda}^{k}(t) e^{-t / x} d t\right|^{m} d x,
\end{aligned}
$$

since

$$
\frac{x^{-k-2}}{\Gamma(k+2)} \int_{0}^{\infty} t^{k+1} e^{-t / x} d t=1
$$

Now

$$
\begin{aligned}
& J \leqq M \int_{0}^{\infty} x^{-m k-2 m-1} d x \mid \int_{0}^{x}\{g(x)-g(t)\} t^{k+1} e^{t / x} d t \\
&-\left.\int_{x}^{\infty}\{g(t)-g(x)\} t^{k+1} e^{-t / x} d t\right|^{m} .
\end{aligned}
$$


By Minkowski's inequality;

$$
J^{1 / m} \leqq J_{1}^{1 / m}+J_{2}^{1 / m}
$$

where

$$
J_{1}=M\left\{\int_{0}^{\infty} x^{-m k-2 m-1}\left|\int_{0}^{x} t^{k+1} e^{-t / x} d t \int_{t}^{x} d g(u)\right|^{m} d x\right\}
$$

and

$$
J_{2}=M\left\{\int_{0}^{\infty} x^{-m k-2 m-1}\left|\int_{x}^{\infty} t^{k+1} e^{-t / x} d t \int_{x}^{t} d g(u)\right|^{m} d x\right\} .
$$

We will show that $J_{1}$ is finite. Putting $t=u y$ in the $t$-integral of $J_{1}$, we get

$$
\begin{aligned}
J_{1} & \leqq M \int_{0}^{\infty} x^{-m k-2 m-1} d x\left\{\int_{0}^{x} u^{k+2}|d g(u)| \int_{0}^{1} y^{k+1} e^{-u y / x} d y\right\}^{m} \\
& \leqq M \int_{0}^{\infty} x^{-m k-m-1} d x\left\{\int_{0}^{x} u^{k+1}|d g(u)|\right\}^{m} .
\end{aligned}
$$

Applying Hölder's inequality to the $u$-integral, we get

$$
\begin{aligned}
J_{1} & \leqq M \int_{0}^{\infty} x^{-m k-2} d x \int_{0}^{x} u^{m k+m}|d g(u)|^{m} \\
& =M \int_{0}^{\infty} u^{m k+m}|d g(u)|^{m} \int_{u^{\prime}}^{\infty} x^{-m k-2} d x \\
& =M \int_{0}^{\infty} u^{m-1}|d g(u)|^{m} \\
& <\infty
\end{aligned}
$$

by hypothesis (ii). We will now show that $J_{2}$ is finite.

Case (a). Let $m>1$. Putting $t=u y$ in the $t$-integral of $J_{2}$, we get

$$
J_{2} \leqq M \int_{0}^{\infty} x^{-m k-2 m-1} d x\left\{\int_{x}^{\infty} u^{k+2}|d g(u)| \int_{1}^{\infty} y^{k+1} e^{-u y / x} d y\right\}^{m} .
$$

Since

$$
\int_{1}^{\infty} y^{k+1} e^{-u y / x} d y<e^{1-u / x} \int_{1}^{\infty} y^{k+1} e^{-y} d y=M e^{-u / x},
$$

we have

$$
J_{2} \leqq M \int_{0}^{\infty} x^{-m k-2 m-1} d x\left\{\int_{x}^{\infty} u^{k+2} e^{-u / x}|d g(u)|\right\}^{m} .
$$


Applying Hölder's inequality, we obtain with $1 / m+1 / m^{\prime}=1$,

$$
\begin{aligned}
J_{2} & \leqq M \int_{0}^{\infty} x^{-m k-2 m-1} d x\left\{\int_{x}^{\infty}|d g(u)|^{m}\right\}\left\{\int_{x}^{\infty} u^{k m^{\prime}+2 m^{\prime}} e^{-m^{\prime} u / x} d u\right\}^{m-1} \\
& \leqq M \int_{0}^{\infty} x^{-m k-2 m-1} d x\left\{\int_{x}^{\infty}|d g(u)|^{m}\right\}\left\{\int_{0}^{\infty} u^{k m^{\prime}+2 m^{\prime}} e^{-m^{\prime} u / x} d u\right\}^{m-1} \\
& =M \int_{0}^{\infty} x^{m-2} d x \int_{x}^{\infty}|d g(u)|^{m} \\
& =M \int_{0}^{\infty}|d g(u)|^{m} \int_{0}^{u} x^{m-2} d x \\
& =M \int_{0}^{\infty} u^{m-1}|d g(u)|^{m} \\
& <\infty
\end{aligned}
$$

by hypothesis (ii).

Case (b). Let $m=1$. We have

$$
\begin{aligned}
J_{2} & \leqq M \int_{0}^{\infty} x^{-k-3} d x \int_{x}^{\infty} u^{k+2} e^{-u / x}|d g(u)| \\
& =M \int_{0}^{\infty} u^{k+2}|d g(u)| \int_{0}^{u} x^{-k-3} e^{-u / x} d x \\
& =M \int_{0}^{\infty}|d g(u)| \int_{1}^{\infty} 2^{k+1} e^{-v} d v \\
& =M \int_{0}^{\infty}|d g(u)| \\
& <\infty,
\end{aligned}
$$

by hypothesis (ii). This completes the proof of Theorem 1 .

Proof of TheOREM 2. The first part of the theorem follows from the sufficiency part of Theorem 1 with $k=0$. The necessity of condition (i) follows from the remark following Lemma 3 . The necessity of condition (ii) is obvious.

In the following theorem, we prove the Abelian relationship between the summability $|R, \lambda, k|_{m}$ and the summability $|A, \lambda|_{m}$ of $\sum a_{n}$.

TheOREм 3. If (i) $\sum a_{n}$ is summable $|R, \lambda, k|_{m}, m \geqq 1, k>1-1 / m$, and (ii) the series $\sum a_{n} \exp \left[-\lambda_{n} x\right]$ converges for all $x>0$ to the sum $f(x)$, then (iii) $\sum a_{n}$ is summable $|A, \lambda|_{m}$. 
Proof. In virtue of (ii),

$$
f^{\prime}(x)=-\frac{x^{k+1}}{\Gamma(k+1)} \int_{0}^{\infty} B_{\lambda}^{k}(t) e^{-x t} d t .
$$

Thus

$$
\begin{aligned}
& \int_{0}^{\infty}\left(1-e^{-x}\right)^{m-1}\left|f^{\prime}(x)\right|^{m} d x \\
& \leqq M \int_{0}^{\infty}\left(1-e^{-x}\right)^{m-1} x^{m k+m} d x\left\{\int_{0}^{\infty}\left|B_{\lambda}^{k}(t)\right|^{m} e^{-x t} d t\right\}\left\{\int_{0}^{\infty} e^{-x t} d t\right\}^{m-1} \\
& =M \int_{0}^{\infty}\left|B_{\lambda}^{k}(t)\right|^{m} d t \int_{0}^{\infty}\left(\frac{1-e^{-x}}{x}\right)^{m-1} x^{m k+m} e^{-x t} d t \\
& \leqq M \int_{0}^{\infty} t^{-m-m k-1}\left|B_{\lambda}^{k}(t)\right|^{m} d t \\
& <\infty
\end{aligned}
$$

by Lemmas 1 and 3 .

In the special case $\lambda_{n}=n$, it follows from results of Borwein [1] and Flett [4] that (i) implies (iii); so that, in this case, (i) alone implies (iii), but this is probably not true for more general $\lambda_{n}$.

\section{REFERENCES}

1. D. Borwein, An extension of a theorem on the equivalence between absolute Rieszian and absolute Cesdro summability, Proc. Glasgow Math. Assoc. 4 (1959) 81-83.

2. K. Chandrasekhran and S. Minakshisundram, Typical means, Oxford Univ. Press, Oxford, 1952.

3. T. M. Flett, Some generalizations of Tauber's second theorem, Quart. J. Math. Oxford Ser. II 10 (1959), 70-80.

4. - On an extension of absolute summability and some theorems of Littlewood and Paley, Proc. London Math. Soc. (3) 7 (1957), 113-141.

5. J. M. Hyslop, On the absolute summability of series by Rieszian means, Proc. Edinburgh Math. Soc. (2) 5 (1936), 46-54.

6. - A Tauberian theorem for absolute summability, J. London Math. Soc. 12 (1937), 176-180.

7. J. S. Ratti, On high indices theorems, Proc. Amer. Math. Soc. 17 (1966), 10011006.

8. D. Waterman, On some high indices theorems, Trans. Amer. Math. Soc. 69 (1950), 468-478.

9. A. Zygmund, On certain integrals, Trans. Amer. Math. Soc. 55 (1944), 170-204.

Wayne State University and

OAKLAND UNIVERSITY 